Title: The Deviance in Deviant Causal Chains

Author: Neil McDonnell

\begin{abstract}
:
Causal theories of action, perception and knowledge are each beset by problems of so-called 'deviant' causal chains. For each such theory, counterexamples are formed using odd or co-incidental causal chains to establish that the theory is committed to unpalatable claims about some intentional action, about a case of veridical perception or about the acquisition of genuine knowledge. In this paper I will argue that three well-known examples of a deviant causal chain have something in common: they each violate Yablos proportionality constraint on causation. I will argue that this constraint provides the key to saving causal theories from deviant chains.
\end{abstract}

Keywords: causation; deviant; causal chain; proportionality; Yablo; causal theory; action; perception; knowledge. 


\title{
The Deviance in Deviant Causal Chains
}

\author{
Neil McDonnell
}

Causal theories of action, perception and knowledge are each beset by problems of so-called 'deviant' causal chains. For each such theory, counterexamples are formed using odd or co-incidental causal chains to establish that the theory is committed to unpalatable claims about some intentional action, about a case of veridical perception or about the acquisition of genuine knowledge. Unless the theories in question can establish a principled reason to reject all and only the deviant chains, the theories will have failed to successfully account for the phenomenon under consideration. I know of no successful attempt to identify what is deviant about the causation in these chains.

In this paper I will argue that three well-known examples of a deviant causal chain have something in common: they each violate a proportionality constraint on causation. I will start by introducing the proportionality constraint from Yablo (1992) and then I will show how that constraint applies to the examples and briefly discuss why we can expect this to generalise. I will then raise a worry about whether we should accept this constraint in general but I will conclude those seeking to utilise the insight about proportionality need not take a stand on this controversial point. 


\section{Proportionality}

The notion of proportionality in causation was introduced by Yablo (1992) and features heavily in his subsequent work ((1997), (2003), (2005)). It has also gained recent prominence in the work of Menzies and List (2010),Sartorio (2010) and Weslake (2013). ${ }^{1}$ The core idea is that a cause is proportional to its effect if and only if there is no more general specification of the event that would have sufficed for the effect (no more determinable specification), and there is no more precise specification of the cause (no more determinate specification) that is required. ${ }^{2}$ This becomes clearer with a concrete, and classic, example from Yablo.

Sophie the pigeon is trained to peck all and only red things.

When a scarlet triangle is placed in front of Sophie, she pecks.

What caused Sophie the pigeon to peck?

There are two answers that one might consider in this case: one could cite the scarlet colour of the triangle, or its redness. Yablo points out that taking the scarlet nature of the triangle to be the cause implies, wrongly, that a crimson triangle would not have worked instead. Citing the redness of the triangle does not imply this and so, whilst more general, makes for a less misleading causal claim. The redness of the triangle is sufficient to explain the pecking.

\footnotetext{
${ }^{1}$ It is worth noting that the proportionality constraint is not motivated by, or even applied to, cases of deviant causal chains in these works.

${ }^{2}$ I stick to talk of the specification of the event to remain neutral on whether a different specification amounts to referring to a different event. Those with a fine-grained conception of events may think that it does, and those with a coarse-grained view will insist that it does not. For further discussion of this issue, and its consequences, see (Author) and (Author).
} 
Of course, we could also say that it was the placing of a coloured triangle that caused Sophie to peck. The triangle was indeed coloured, but a blue or green triangle would not have elicited a peck and so it is plausible that citing the coloured nature of the triangle will give a false impression about those cases. To avoid such a false impression we should specify the feature of the triangle which was actually required for the pecking: that it was red.

So, the redness of the triangle was both sufficient and required for Sophie's pecking. This is what makes the redness of the triangle the proportional cause of the pecking.

In an alternative story, also popular in the literature, a second pigeon, Alice, is trained to peck all and only scarlet things. When a scarlet triangle is placed in front of Alice, she of course pecks too. In this case the redness of the triangle is required, but not sufficient, for the peck: citing the redness may give the false impression that my crimson triangle would elicit a peck too. Thus in this case the scarlet colour of the triangle is the proportional cause of the pecking.

Notice that in both Sophie and Alice's case, specifying that it was a triangle (rather than a square or circle or merely a patch) also amounts to giving unnecessary detail. Any shape would do, and so, for a fully proportional specification of what caused Sophie to peck, we ought to say that it was the placing of a red shape in front of her. This demonstrates that proportionality is not just about one dimension of specification - i.e. colour - but all aspects of the event specification.

For Yablo, causation is subject to a proportionality constraint such that only proportional causal connections are genuine. Whilst I will return to 
question the role of this constraint in $\S 3$, I will adopt this view for the time being and consider how it might impact on the issues surrounding so-called deviant causal chains. ${ }^{3}$

\section{Deviance}

An ordinary causal chain links some initial event or property, via one or more intermediates, to some end state or event in such a way that the first is considered a cause of the last via that chain. Such chains are taken to be absolutely commonplace and a straightforward feature of the world we live in, as when we tip the first domino in a run and thereby cause the last to fall. Some have gone further and claimed that such chains reveal the transitivity of the causal relation, though this is now a controversial notion. ${ }^{4}$ What makes some cases apparently 'deviant' is the fact that this chaining reveals a causal connection which, when combined with a given theory of action, perception or knowledge, yields a counter-intuitive conclusion. In each case there is something peculiar or unexpected about the chain in question and it is that peculiarity which is the source of the theory-confounding example. It will be my contention in this paper that the "deviance" in the case of deviant causal chains is the fact that at least one step in the chain violates the proportionality constraint just introduced.

The first example of such a chain that I will consider is from Peacocke and

\footnotetext{
${ }^{3}$ Full details of how Yablo defines the proportionality constraint can be found in Yablo (1992). Alternative specifications can be found in (Author), Sartorio (2010) and Weslake (2013). For the purposes of remaining neutral on related issues, I do not commit to any given specification. If what I say here follows from the intuitive notion, that should help frame the requirements for a tighter specification.

${ }^{4}$ See McDermott (1995), Ehring (1987), Hall (2000), (Author) and Paul (2000).
} 
concerns causal theories of perception which hold that to perceive a thing is to be causally related to that thing in the right sort of way. Examples such as the following put pressure on how that right sort of way might be spelled out:

...consider for instance the case of a man who with his eyes open but under the influence of a hallucinogen is surrounded by redwood trees that produce a scent that causes him to have a vivid visual image of redwood trees which happens precisely to match his surroundings. (Peacocke, 1979, p.123)

The thought here is that the redwood trees cause the subject to have a vivid visual image that matches those redwood trees, just as would happen in ordinary perception, but in this case the causal chain runs via a scent and a hallucinogen. No viable theory should call this a case of veridical perception yet standard causal theories of perception do not have the resources to say what makes the chain 'deviant' and why this doesn't count as a case of perception. Therefore, this case is a counterexample to those causal theories of perception.

Most attempts to respond to this counterexample accept the causal status of the connection between the redwoods and the visual image and move on to refining or abandoning the theory which takes that connection to constitute perception. ${ }^{5}$ However, by applying the proportionality constraint that Yablo has introduced, I think we can see that it is the causal status of the chain that is where the problem lies.

\footnotetext{
${ }^{5}$ cf Mitchell (1982).
} 
Holding fixed the presence of the hallucinogen, the scent is what gives rise to the visual image which precisely matches the surroundings. However that precise arrangements of redwoods is not a proportional cause of the scent-many other arrangements or even a synthetic scent would do the job-and so is not a proportional link in the causal chain. That precise image was not caused by that precise arrangement of redwoods but rather was caused by there being some redwood scent present at all. Peacocke's counterexample requires that there is a perfect match between the scene and the visual image and it requires that the former causes the latter but by the lights of the proportionality constraint it is not that precise arrangement of redwoods that cause the image, but merely whatever arrangement would produce that scent. So if we adopt a proportionality constraint on causation, the counterexample fails.

Turning now to an example from Davidson which concerns causal theories of intentional action. On such theories an event is an instance of intentional agency if it is (at least partly) caused by a corresponding mental state within an agent. The following example is supposed to demonstrate that some further constraint is required:

A climber might want to rid himself of the weight and danger of holding another man on a rope, and he might know that by loosening his hold on the rope he could rid himself of the weight and danger. This belief and want might so unnerve him as to cause him to loosen his hold, and yet it might be the case that he never chose to loosen his hold, nor did he do it intentionally. 
(Davidson, 1980, p.79)

Here it appears as though the climber's mental state - the desire to to be safer and the knowledge that loosening his grip would achieve this-causes him to loosen his grip, but it does so via an unintentional step. The mental state caused a corresponding action and so it can be said to demonstrate agency, on a causal theory of action. However the event was clearly lacking the sort of intent ${ }^{6}$ required for genuine agency, and yet the causal theory of action does not have the resources to say why this causal chain is to be ruled out, why it is 'deviant'. Therefore, this case is a counterexample to such a causal theory of action.

Once again, commentators typically accept the causal connection between the mental state and the action and get on with refining or abandoning the theory which takes that connection to constitute action. ${ }^{7}$ However, once again, applying the proportionality constraint on causation suggests that the causal connection is where the problem arises.

We can grant that the nervousness caused the rope to slip but ask: what caused the nervousness? In this instance the thoughts of letting the rope slip may be the thoughts that caused the nervousness, but they are only the proportional cause if there is no more general specification of that event that would have caused the same state. What about such beliefs and wants are unnerving, we might wonder? It is surely nothing to do with ropes or weight

\footnotetext{
${ }^{6}$ We can simply stipulate that this is not some regular routine the climber goes through in full knowledge of the outcome. Such distinct cases do seem to demonstrate agency, as Tannjso (2009) argues.

${ }^{7}$ Witness the to-and-fro on this point between Schlosser (2007) and Tännsjö (2009) in Analysis.
} 
or grip per se, which are the given content of the thought, but rather it is the fact that in this context that content belongs to a broader type of thought, of wilfully harming someone else, and the consequences of such. It is that determinable type of thought, concerning harm and selfishness, and not the determinate sort, concerning letting a rope slip, that is unnerving. As such it is these determinable thoughts which serve as the proportional cause of the nervousness, not the overly specific, determinate, thoughts regarding ropes and slippage. By invoking the proportionality constraint we can insist that it was the thoughts of harming someone else at all that caused the slippage and, without the additional detail concerning ropes etc., this thought will not appropriately match the outcome in the manner required to be a genuine counterexample. Once again the initial cause has been overly specified to create the problem.

Finally, consider the following epistemic example: ${ }^{8}$

Steve is in Japan and finds a newspaper that has fallen from the a vendor's stand. The headline reads: Earthquake in Japan! Steve forms the belief that there has been a recent earthquake in Japan, and this belief is true, however unbeknownst to Steve the newspaper is 30 years old. Nevertheless the earthquake that occurred today was what dislodged the ancient newspaper.

In this case Steve forms a true belief about there being a recent earthquake and this belief seems to have been caused, albeit indirectly, by that recent earthquake. According to the causal theory of knowledge defended

${ }^{8}$ I take this example from John Preston, who informs me via personal correspondence that he took it from unpublished work by Michelle Forster circa 1995. 
by Goldman in (1967), this ought to count as a case of knowledge. However, it is obvious that Steve's belief lacked justification - a 30 year old newspaper cannot report yesterday's events - and his resultant belief does not seem to constitute a case of knowledge. Therefore, this is a counterexample to such a causal theory of knowledge.

One again the causal chain that connects the earthquake and the belief that there was an earthquake is peculiar to say the least. Had any current paper fallen from the rack, the belief would not have formed and had the earthquake not taken place, but the newspaper still caught Steve's attention, the belief which formed would have been false. Granting that the reading of the paper caused the belief to form, we can then ask: what caused Steve to read the paper? We know that in the actual case that the earthquake dislodged the paper, but this does not make the earthquake a proportional cause of Steve's reading it. Had it been dislodged any other way, Steve would still have spotted and read the paper and gone on to form the belief. This means that citing the earthquake as the cause of the belief is ruled out in favour of citing the dislodging of the newspaper. Once again, this means that there is not a causal connection between the earthquake and the belief about the earthquake, but rather there is a causal connection between the dislodging of the newspaper and the belief that there was an earthquake. The former is required to form the counterexample and the latter is benign with respect to Goldman's causal theory.

The deviance cases are not unlike the case of Alice the pigeon. We know that Alice is trained to peck all and only scarlet things. Now, imagine that a red triangle is placed in front of her. In this case Alice pecks, and someone 
witnessing this might wrongly think that all red things (or all triangles) will cause Alice to peck. But it is just a coincidence - a fragile fluke - that the shade of red on this occasion was the right one. To draw a robust conclusion about redness in general would be unwarranted and would mean you were misled by the coincidence. In the case of the redwood hallucination, the exact match with the environment is just such a fragile fluke, and the presence of an appropriate scent (in combination with the hallucinogen) is the robust cause of the pictured scene. In the case of the climber, that the precise form of the thought matches the subsequent action is also a fluke since any other source for that same nervousness would have brought about the same effect, and in the case of the earthquake the fluke was that the old headline, in the manner of a stopped clock, mapped onto the current facts despite being unconnected to them. In each case the proportionality constraint rules out the event being a cause when it is specified in the way that made it a fluke, and rules in the event as being a cause when it is specified in the way that makes it robust. Thus, the proportionality constraint identifies what is deviant about these deviant causal chains and motivates denying the counterexamples as a result. Perhaps it was a causal problem all along.

\section{The Role of Proportionality}

I have introduced the proportionality constraint as a constraint on causation itself, and shown that in the examples given this constraint allows us to see what is deviant about so-called deviant causal chains. This is a sub- 
stantive claim about causation, rather than about causal theories and so is not specific to any given causal theory of some phenomenon. However there remains something of a puzzle about the proportionality constraint as it presently stands: is it a semantic constraint on which causal claims are true, or merely a pragmatic constraint on which are acceptable to assert?

One reason to think that proportionality is not a semantic constraint on causation is the simple fact that we would ordinarily accept many out-ofproportion causal claims as true: drinking hemlock caused Socrates to die, taking valium caused me to sleep and killing Oswald caused Jack Ruby to be arrested. These are out of proportion because many poisons, not just hemlock, would have killed Socrates, just as many other sleeping pills would have worked in place of valium to get me the rest I needed, and if Ruby had killed anyone in the manner that he did he would have been arrested. Thus there is a more general specification of the event that can be cited in each case in place of the one we ordinarily would, and yet we stick to supplying the more detailed information when we have it. If proportionality is a semantic constraint on causation, much of our ordinary causal talk is not just infelicitous, but outright false because of this over-specification. This seems implausible.

On the other hand we might think that a mere pragmatic constraint on causation leaves the deviant chains as causal, just not maximally informative or explanatory. ${ }^{9}$ Whilst this rescues much of ordinary causal talk, it leaves the door open to the counterexamples: whilst there is a more felicitous way

\footnotetext{
${ }^{9}$ Weslake (2013) makes an interesting, and plausible, case to the effect that proportionality is a dimension of explanatory virtue. This would render it a pragmatic rather than semantic constraint on causation itself.
} 
of telling the causal story in the counterexamples discussed here, there is nevertheless a true telling of the same story that creates a problem for the causal theories of action, perception and knowledge. The counterexamples only require that the component causal claims are true, not that they are optimally formed.

Whilst I think there is a genuine dilemma to be faced in the philosophy of causation when it comes to proportionality, I do not think that it needs to be settled before the defender of such causal theories of action, perception or knowledge can make use of the insight claimed in this paper. The basic challenge such a theorist faces is to say what is deviant about the counterexamples so that they can be parcelled out without loss to their theory, they do not have to say that such cases are not causal. When specifying what sorts of causal connection are required to constitute perception or action or knowledge, defenders of a causal theory need only add a clause which states that the causal connections in question be proportional. No broader commitment to the semantic/pragmatic status of proportionality in causation is required.

\section{Conclusion}

This paper has argued that certain prominent examples of so-called 'deviant' causal chains violate the independently plausible proportionality constraint on causation. If this generalises then causal theories of any given phenomena can rule out the unwanted commitments that deviant chains impose by simply embracing a proportionality constraint on which causal relations the 
theory admits. The further question of whether proportionality is a semantic or a pragmatic constraint on causation remains pressing for the philosopher of causation, but it need not hold back progress elsewhere. Those wishing to posit a causal theory of some phenomenon do not need to revise the philosophy of causation to find the deviance in deviant causal chains.

\section{References}

Davidson, D. (1980). Freedom to act. In Essays on Action and Events, pages 63-82. Oxford University Press.

Ehring, D. (1987). Causal relata. Synthese, 73(2):319-328.

Goldman, A. I. (1967). A causal theory of knowing. Journal of Philosophy, 64(12):357-372.

Hall, N. (2000). Causation and the price of transitivity. Journal of Philosophy, 97(4):198-222.

McDermott, M. (1995). Redundant causation. British Journal for the Philosophy of Science, 46(4):523-544.

Menzies, P. and List, C. (2010). The causal autonomy of the special sciences. In McDonald, C. and McDonald, G., editors, Emergence in Mind. Oxford: Oxford University Press.

Mitchell, D. (1982). Deviant causal chains. American Philosophical Quarterly, 19(4):351-353.

Paul, L. A. (2000). Aspect causation. Journal of Philosophy, 97(4):235-256. 
Peacocke, C. (1979). Deviant causal chains. Midwest Studies In Philosophy, $4(1): 123-155$.

Sartorio, C. (2010). The prince of wales problem for counterfactual theories of causation. In Hazlett, A., editor, New Waves in Metaphysics, pages 259-276. Palgrave McMillan, New York.

Schlosser, M. E. (2007). Basic deviance reconsidered. Analysis, 67(295):186194.

Tännsjö, T. (2009). On deviant causal chains - no need for a general criterion. Analysis, 69(3):469-473.

Weslake, B. (2013). Proportionality, contrast and explanation. Australasian Journal of Philosophy, 91(4):785-797.

Yablo, S. (1992). Mental causation. Philosophical Review, 101(2):245-280.

Yablo, S. (1997). Wide causation. Philosophical Perspectives, 11(11):251281.

Yablo, S. (2003). Causal relevance. Philosophical Issues, 13(1):316-28.

Yablo, S. (2005). Prime causation. Philosophy and Phenomenological Research, 70(2):459-467. 